\title{
Charcoal Is a Sensitive, Specific, and Stable Marker for the Diagnosis of Aspiration in Hamsters
}

\author{
AVRAHAM AVITAL, SHERMAN YOAV, AND CHAIM SPRINGER \\ Institute of Pulmonology [A.A., C.S.] and Department of Pathology [S.Y.], Hadassah University Hospital \\ and the Hadassah-Hebrew University Medical School, Jerusalem, Israel
}

\begin{abstract}
ABST
The diagnosis of recurrent aspiration in young children is a
perpetual challenge because there is no specific gold standard test
to be used. The finding of lipid-laden alveolar macrophages in
bronchoalveolar lavage (BAL) is a sensitive but nonspecific
marker for the diagnosis of aspiration. We conducted a short-
term study comparing tracheal instillation of saline, milk, or a
milk-charcoal mixture in hamsters. BAL cytology, lipid-laden
alveolar macrophage index, charcoal alveolar macrophage index,
and lung histology were monitored for $10 \mathrm{~d}$. A long-term study
was performed, and hamsters were monitored for $92 \mathrm{~d}$ after
milk-charcoal tracheal instillation. Baseline animals $(n=4)$ had
no tracheal instillation. Saline- and milk-instilled animals had
BAL performed after 1 ( $n=4), 3(n=4)$, and $10(n=4) \mathrm{d}$.
Milk-charcoal-instilled animals had BAL performed after $1(n=$
4), 3 ( $n=4), 10(n=4), 30(n=2), 58(n=2)$, and $92(n=$
2) d after tracheal instillation. Total cell counts and percent
neutrophils in BAL fluid increased significantly and similarly
after milk and milk-charcoal instillation on d 1 compared with
\end{abstract}
The diagnosis of recurrent aspiration pneumonia in young children is a perpetual challenge because there is no gold standard test sufficiently specific for the diagnosis. Barium swallow with videofluoroscopy and radionuclide milk scintigraphy (milk scan) are neither sensitive nor specific for the diagnosis of aspiration $(1,2)$. LLAM in BAL has been found to be a sensitive but nonspecific test in children and adults (3, 4). Recently, Elidemir et al. (5) described a novel diagnostic method for aspiration in a murine model. They stained milk proteins ( $\beta$-lactalbumin and $\beta$-lactoglobulin) in alveolar macrophages by an immunocytochemical method. They found that their method is very sensitive and very specific compared with oil red $\mathrm{O}$ staining, but their method was shown to be positive only for the 3-4 d after induced aspiration. Aspiration can occur from above as in children with neurologic impairment,

Received June 25, 2001; accepted November 8, 2001

Correspondence and reprint requests: Avraham Avital, M.D., Institute of Pulmonology, Hadassah University Hospital, POB 12000, Jerusalem 91120, Israel; e-mail: avi@lung.hadassah.org.il baseline and saline-instilled animals. Lipid-laden alveolar macrophage index increased significantly only on d 3 after milk and milk-charcoal instillation compared with all days in the salineinstillation group. Charcoal alveolar macrophage index increased significantly after milk-charcoal instillation (d 1-58) from baseline or all days in the saline-instillation group. We conclude that charcoal particles instilled in tracheas of hamsters can be easily identified in BAL fluid and in lung parenchyma for as long as 3 mo after a single instillation and could potentially be used as a sensitive, specific, and stable marker for the diagnosis of aspiration, although the issue of its applicability to humans is still unsolved. (Pediatr Res 51: 397-401, 2002)
BAL, bronchoalveolar lavage

\section{Abbreviations}
ChAM, charcoal alveolar macrophage
LLAM, lipid-laden alveolar macrophage

but can be secondary to gastroesophageal reflux and may occur only during the massive episodes and not necessarily every day. It is therefore important to have a sensitive and specific marker that could help diagnose aspiration that may have occurred days or weeks previously. This marker should be made from an inert nonharmful material that can be given orally with food, is not produced endogenously, can be swallowed by alveolar macrophages, and can easily be identified in BAL fluid and alveolar macrophages for a substantial period of time. We therefore performed tracheal instillation of activated charcoal particles mixed with milk, compared it with instillation of normal saline or milk in hamsters, and monitored the animals for BAL cytology, LLAM index, and ChAM index during the next 3 mo.

\section{METHODS}

Animals. Forty-six female Syrian hamsters (Mesocricetus auratus), 9-12 wk old, weighing 100-140 g, and obtained from Harlan Sprague-Dawley (Indianapolis, IN, U.S.A.), were 
used for the experiments. The study was approved by the local Institutional Animal Care and Use Committee. Four hamsters were used as baseline (naïve) controls (without tracheal instillation). Forty-two hamsters had tracheal instillation of $0.1 \mathrm{~mL}$ of either sterile normal saline $(n=12)$, pasteurized 3\% milk $(n$ $=12)$, or a milk-charcoal mixture $(n=18)$ using pure activated charcoal powder (Merck Company, Whitehouse Station, NJ, U.S.A.) $5 \mathrm{mg} / \mathrm{mL}$ within $3 \%$ pasteurized milk. Naive animals were killed and underwent BAL. Saline- and milkinstilled animals were killed and BAL was performed on $\mathrm{d} 1(n$ $=4), 3(n=4)$, and $10(n=4)$ after tracheal instillation. Milk-charcoal-instilled animals underwent BAL on $\mathrm{d} 1(n=$ 4), $3(n=4), 10(n=4), 30(n=2), 58(n=2)$, and $92(n=$ 2) after tracheal instillation. Lungs from all animals were preserved in formaldehyde for further histologic evaluation.

Anesthesia. A mixture of ketamine hydrochloride $(50 \mathrm{mg} /$ $\mathrm{mL}, 10 \mathrm{~mL})$ and droperidol $(2.5 \mathrm{mg} / \mathrm{mL}, 2 \mathrm{~mL})$ was administered by i.p. injection, $0.1-0.2 \mathrm{~mL}$ before intubation and tracheal instillation, and $0.3-0.4 \mathrm{~mL}$ before exsanguination.

Tracheal instillation. Direct intubation was performed with a blunted metal needle. A few ventilations with a small Ambu bag were given to ascertain intratracheal position of the needle and movement of the chest. Normal saline, milk, or milkcharcoal solution $(0.1 \mathrm{~mL})$ was slowly instilled into the trachea, followed by a few ventilations with the Ambu bag to disperse the fluid and to prevent apnea.

Bronchoalveolar lavage. On days when BAL was to be performed, under deep anesthesia exsanguination was performed by transection of the abdominal aorta. The trachea was exposed, and a blunted needle covered with a polyethylene cannula was inserted into the trachea. Three aliquots of $5 \mathrm{~mL}$ of sterile $0.9 \%$ saline were injected and withdrawn with a total recovery of $87 \pm 1.3 \%$. The fluid was examined for total cell counts, and slides for differential counts (using Diff-Quik solution, Baxter Diagnostics AG, Dudingen, Germany) and oil red $\mathrm{O}$ staining were prepared on a Shandon Cytospin 3 (Cheshire, England) using approximately $100 \mu \mathrm{L}$ of BAL fluid.

Lung pathology. Trachea and lungs were excised and fixed with formalin. Hematoxylin-eosin was used for staining.

Oil red $O$ staining. Standard oil red $\mathrm{O}$ staining and hematoxylin counterstain of BAL slides were performed (6).

LLAM index. One hundred macrophages were blindly counted after oil red $\mathrm{O}$ staining, each assigned a grade of $0-4$ according to the criteria of Colombo et al. (7), the LLAM index being the sum, with a potential maximum of 400 . The criteria for LLAM grading were 0 , no red staining; 1 , up to $25 \%$ of the cytoplasm opacified; 2 , up to $50 \%$ opacified; 3 , up to $75 \%$ opacified; and 4, totally opacified.

ChAM index. One hundred unselected macrophages were counted, and each macrophage was counted as positive (1 point) if at least one black charcoal particle was seen in the cytoplasm, the ChAM index being the sum, with a potential maximum of 100 .

Statistics. Group data are expressed as mean \pm SEM. Differences among groups were compared using ANOVA with Bonferroni correction. Differences were taken as significant when $p<0.05$.

\section{RESULTS}

Results of total cell counts, total macrophages, percent neutrophils, and LLAM and ChAM indices of BAL are presented in Table 1 and illustrated in Figures 1 and 2.

Total cell counts. There was a significant increase in total cell counts on $\mathrm{d} 1$ after milk instillation $(p<0.05)$ and milk-charcoal instillation $(p<0.01)$ compared with baseline and saline days (Fig. 1a).

Total macrophages. Total macrophages increased (Table 1) on $\mathrm{d} 1$ after milk $(p<0.01)$ and milk-charcoal $(p<0.05)$ instillation compared with baseline day and on d 92 after milk-charcoal instillation compared with baseline and saline days $(p<0.05)$.

Percent neutrophils. Compared with baseline day, percent neutrophils increased significantly $(p<0.01)$ on $\mathrm{d} 1$ after milk instillation and milk-charcoal instillation d 1, 3, 30, and 58 (Fig. $1 b$ and Table 1). Compared with saline $\mathrm{d} 1$, percent neutrophil counts increased significantly $(p<0.01)$ on $\mathrm{d} 1$ after milk-charcoal instillation (Fig. 1b).

LLAM index. Compared with baseline day, LLAM index increased significantly on $\mathrm{d} 3(p<0.001)$ and $10(p<0.05)$ after milk instillation and on d $3(p<0.001)$ after milkcharcoal instillation. Compared with the postsaline instillation study (Fig. 1c), LLAM index increased significantly only on d 3 after milk $(p<0.05)$ and after milk-charcoal instillation $(p<$ $0.01)$. Within the milk-charcoal study days, LLAM d 3 was

Table 1. Total cell counts, total macrophages, percent neutrophils, LLAM index, and ChAM index in baseline animals and after tracheal instillation of normal saline, milk, and milk-charcoal solution in hamsters

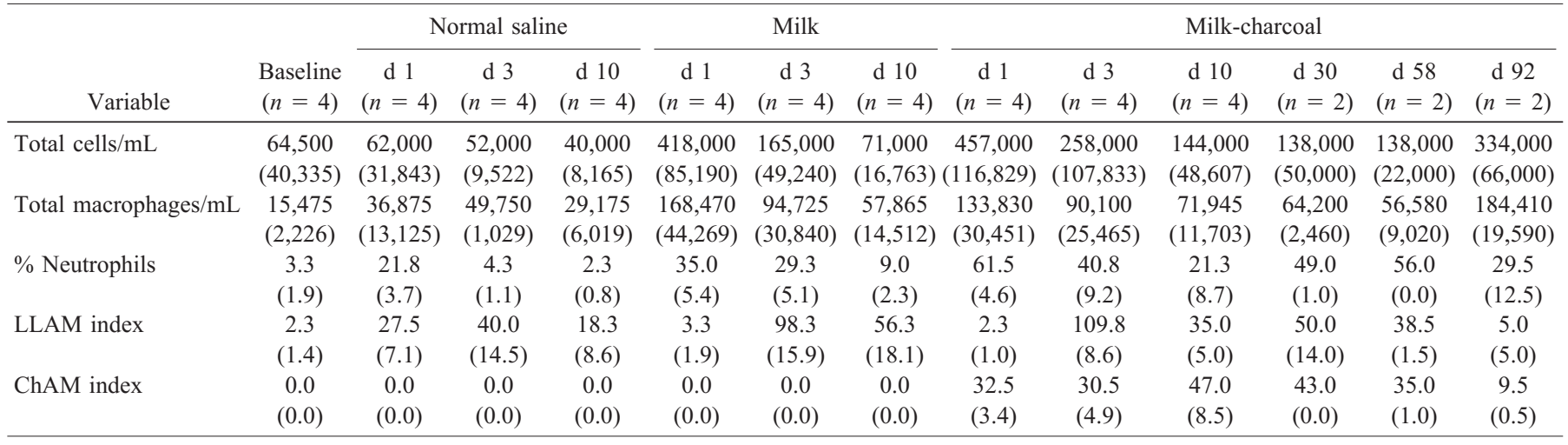




\section{Short term study}
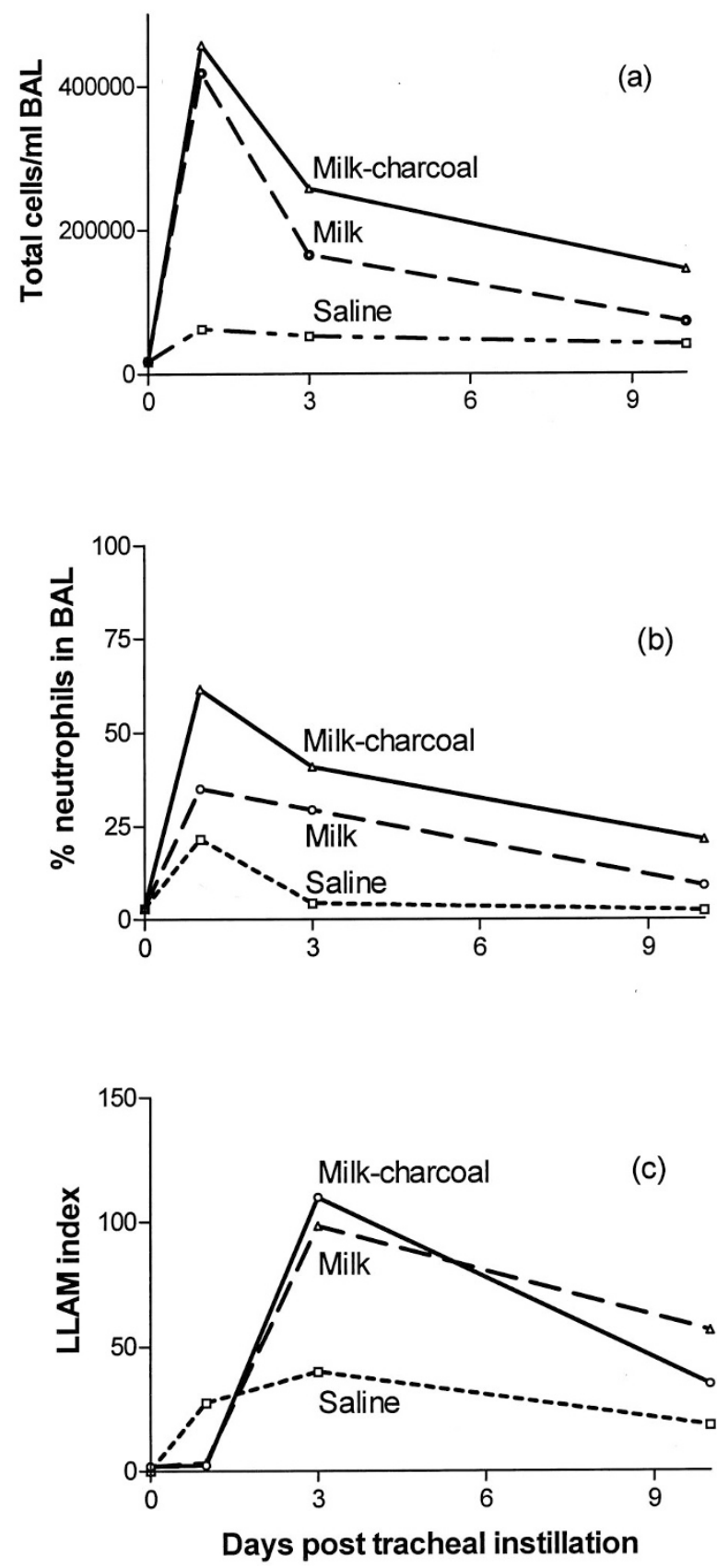

Figure 1. Short-term study of charcoal tracheal instillation in hamsters. Total cells $(a)$, percent neutrophils $(b)$ and lipid LLAM index $(c)$ after a single tracheal instillation of saline, milk, or milk-charcoal solution in hamsters.

significantly higher $(p<0.001)$ than all the other days (Fig. $2 b)$.

ChAM index. Charcoal particles were easily identified from d 1 after milk-charcoal instillation within alveolar macrophages and free in BAL fluid (larger particles) throughout all studied days. No charcoal particles were found in baseline, saline-, or milk-instilled animals. Results of ChAM score are presented in Table 1 and illustrated in Figure 2b. ChAM scores of saline or baseline animals were significantly lower than
Long-term study
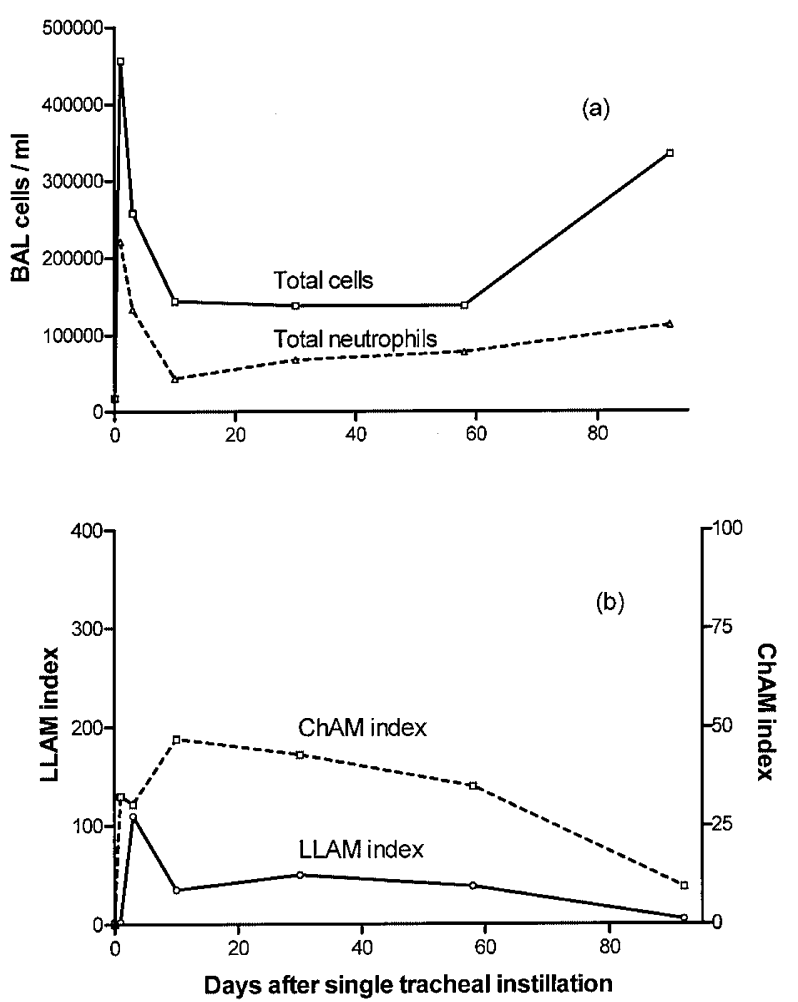

Figure 2. Long-term study of charcoal tracheal instillation in hamsters. Long-term follow-up of total cells, total neutrophils (a), and LLAM and ChAM indices $(b)$ in BAL of hamsters after a single tracheal instillation of milk-charcoal solution.

scores of $\mathrm{d} 1(p<0.01), 3(p<0.01), 10(p<0.001), 30(p$ $<0.01)$, and $58(p<0.05)$ of milk-charcoal instilled animals. On $d 1$, charcoal particles with negative lipid staining after milk-charcoal instillation appeared and are illustrated in Figure 3. Oil red O-stained lipid vacuoles (appearing as gray in black and white illustrations) and charcoal particles are illustrated within alveolar macrophages on d 3 after charcoal-milk instillation (Fig. 4).

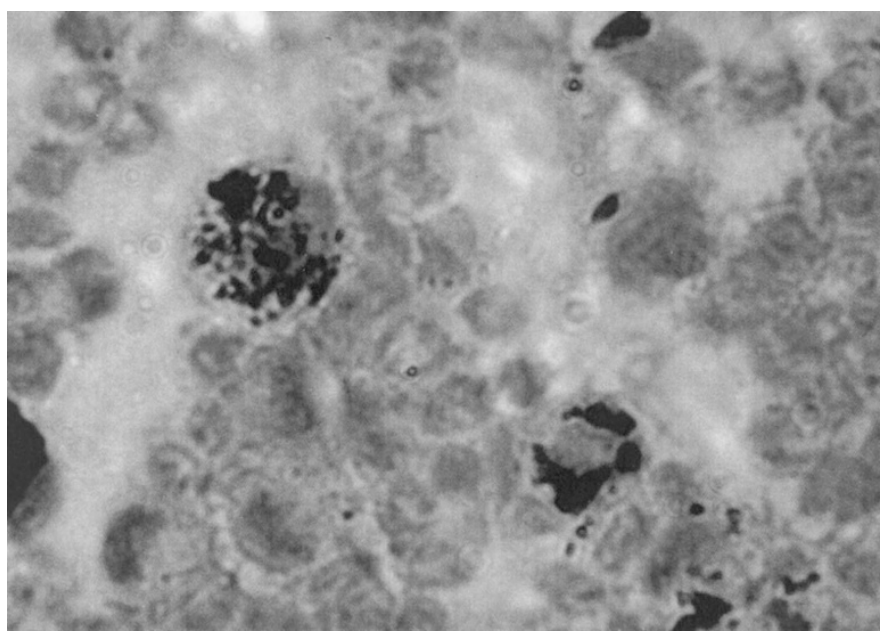

Figure 3. Alveolar macrophages on $d 1$. Alveolar macrophages filled with charcoal (black) particles in BAL cells on d 1 after tracheal instillation of milk-charcoal solution. 


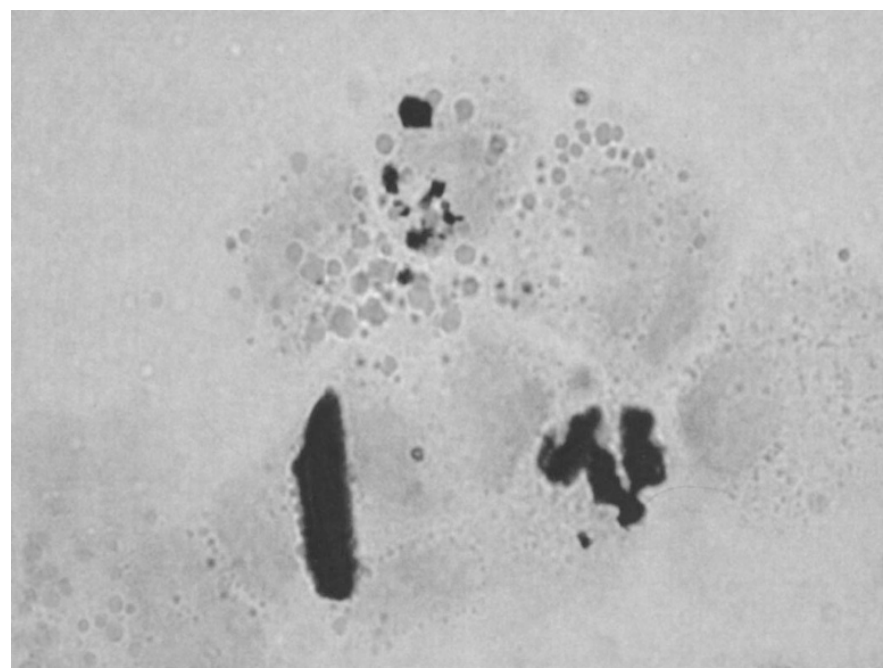

Figure 4. Alveolar macrophages on d 3. Alveolar macrophages filled with lipid vacuoles (gray) and charcoal particles (black) on d 3 after tracheal instillation of milk-charcoal solution.

Lung histology. There was no evidence of parenchymal inflammation of lungs after saline tracheal instillation. There was a mild peribronchial inflammation in the lungs after milk tracheal instillation on $\mathrm{d} 1$ and 3 , returning to normal lung histology on d 10. In milk-charcoal-instilled animals, a mild peribronchial inflammation was found on $\mathrm{d} 1,3$, and 10 . Normal lung anatomy was observed on d 30,58 , and 92 , and charcoal particles could be easily identified in the lung parenchyma, close to bronchioles or within alveolar spaces. Most charcoal particles remaining in the lung parenchyma were of a greater size. On d 58 a few giant cells were found in the lung parenchyma, probably secondary to the foreign body effect of the large particles.

\section{DISCUSSION}

In this study we instilled normal saline, milk, and milk mixed with activated charcoal particles into hamsters' trachea and found a significant increase in the LLAM index only on d 3 in milk- and milk-charcoal-instilled animals compared with saline-instilled animals. Charcoal particles were clearly identified in alveolar macrophages from the first day until $92 \mathrm{~d}$ after the single instillation. The main problem with the LLAM index is that it is not specific for aspiration and that the sensitivity of this test is probably time-dependent. Colombo et al. (7) performed a single milk instillation in rabbits and showed an increase in the LLAM index for the first $2 \mathrm{~d}$ after instillation, starting already $6 \mathrm{~h}$ after milk instillation and returning to normal for the subsequent days. In our study, lipid vacuoles were found within alveolar macrophages only on the third day after tracheal instillation of milk. The difference between the two studies can result from the fact that different animals were used. In a study of induced pneumonia by inhaled pneumococci in different animals (8), the clearance rate of pneumococci in rabbit macrophages was much faster $(94 \%$ clearance rate within $1.5 \mathrm{~h}$ ) than the rate in rats or guinea pigs. It is possible that hamsters clear aspirated fluids at a slower rate than rabbits, although it is not clear why alveolar macrophages from hamsters swallow charcoal on the first day and show lipid-laden vacuoles only on the third day.

The LLAM index has been shown to be a nonspecific marker of aspiration because LLAM can appear in different inflammatory, postinflammatory, or allergic diseases of the lung, in chronic airway diseases, or even after i.v. lipid alimentation in newborn babies (3, 9-11). In our study we could see LLAM even in saline-instilled animals. It seems that increased LLAM staining occurs only during the first few days after tracheal instillation of milk and that the changes during the next days or weeks are probably nonspecific. These changes could be related to the inflammation itself or to the release of lipids from destroyed membranes and surfactant secondary to the infection $(12,13)$.

Activated charcoal has been used in medicine as it is an inert substance with the ability to adsorb a wide variety of substances. It prevents the gastrointestinal absorption of various drugs and toxins and increases their elimination even after systemic absorption $(14,15)$.

Activated charcoal has also been used as a marker for motility and transit time studies of the gastrointestinal tract and for quantitative evaluation of fat excretion in stool. Accidental aspiration of a large amount of activated charcoal with sorbitol administered for the treatment of poisoning induced adult respiratory distress syndrome gradually improved after bronchoscopic removal of charcoal particles and supportive care (16). Other cases of charcoal aspiration induced fatal pulmonary aspiration (17) or bronchiolitis obliterans with air trapping on chest $\mathrm{x}$-rays and large amounts of charcoal identified at postmortem examination (18). The oral or nasogastric dose used in cases of intoxication is $50-100 \mathrm{~g}$ in adults and $1 \mathrm{~g} / \mathrm{kg}$ in children, and the dose may be repeated a few times per day. In our study we used a smaller amount of activated charcoal (5 $\mathrm{mg} / \mathrm{mL}$, correlating to approximately $4 \mathrm{mg} / \mathrm{kg}$ ), and the amount needed for the detection of charcoal particles in alveolar macrophages may even be much smaller. As a matter of fact, for the diagnosis of aspiration we need to detect only one single charcoal particle, but further studies have to be performed to evaluate possible quantitative criteria of aspiration.

In our study, we used milk mixed with a small amount of activated charcoal particles to compare the ChAM index with the traditional LLAM index in hamsters. Charcoal particles within alveolar macrophages or free in BAL fluid were indeed found in all the studied days, whereas the LLAM index was significantly different from saline instillation only on $\mathrm{d} 3$. The profile of BAL cells and the peribronchial inflammation found in the lungs of the hamsters were similar in the milk- and milk-charcoal-instilled animals, supporting the theory that charcoal itself is an inert material and does not induce a different pattern of inflammation. It seems that charcoal particles could potentially be used as a reliable and nonharmful marker for aspiration in humans, although the applicability of this marker for young children suspected to suffer from recurrent aspiration is meanwhile limited. Our reservations are related to the increase in total BAL cells found 3 mo after a single milk-charcoal instillation (Fig. 2a). This hypercellularity in BAL may be explained by the fact that charcoal particles included large particles (up to $25 \mu \mathrm{m}$ ), which may remain 
within the lung tissue for extended periods and behave as foreign bodies, inducing a chronic inflammatory response. Before any attempt is made to use this method in humans, further safety studies with more animals, smaller doses of charcoal, and smaller or uniform charcoal particles should be performed.

\section{REFERENCES}

1. Heyman S, Kirkpatrick JA, Winter HS, Treves S 1979 An improved radionuclide method for the diagnosis of gastroesophageal reflux and aspiration in children (milk scan). Radiology 131:479-482

2. McVeagh P, Howman-Giles R, Kemp A 1987 Pulmonary aspiration studied by radionuclide milk scanning and barium swallow roentgenography. Am J Dis Child 141:917-921

3. Corwin RW, Irwin RS 1985 The lipid-laden alveolar macrophage as a marker of aspiration in parenchymal lung disease. Am Rev Respir Dis 132:576-581

4. Langston C, Pappin A 1996 Lipid-laden alveolar macrophages as an indicator of aspiration pneumonia. Arch Pathol Lab Med 120:326-327

5. Elidemir O, Fan LL, Colasurdo N 2000 A novel diagnostic method for pulmonary aspiration in a murine model: immunocytochemical staining of milk proteins in alveolar macrophages. Am J Respir Crit Care Med 161:622-626

6. Mallory FB 1942 Pathological Technique. WB Saunders Co, Philadelphia, p 118
7. Colombo JL, Hallberg TK, Sammut PH 1992 Time course of lipid-laden pulmonary macrophages with acute and recurrent milk aspiration in rabbits. Pediatr Pulmonol 12:95-98

8. Coonrod JD, Varble R, Jarrels MC 1990 Species variation in the mechanism of killing of inhaled pneumococci. J Lab Clin Med 116:354-362

9. Knauer-Fischer S, Ratjen F 1999 Lipid-laden macrophages in bronchoalveolar lavage fluid as a marker for pulmonary aspiration. Pediatr Pulmonol 27:419-422

10. Recalde AL, Nickerson BG, Vegas M, Scott CB, Landing DH, Warburton D 1984 Lipid-laden macrophages in tracheal aspirates of newborn infants receiving intravenous lipid infusions: a cytologic study. Pediatr Pathol 2:25-34

11. Kajetanowicz A, Stinson D, Laybolt KS, Resch L 1999 Lipid-laden macrophages in the tracheal aspirate of ventilated neonates receiving intralipid: a pilot study. Pediatr Pulmonol 28:101-108

12. Cohen AB, Cline MJ 1972 In vitro studies of the foamy macrophage of postobstructive endogenous lipoid pneumonia in man. Am Rev Respir Dis 106:69-78

13. Desai R, Tetley TD, Curtis CG, Powell GM, Richards RJ 1978 Studies on the fate of pulmonary surfactant in the lung. Biochem J 176:455-462

14. Neunoven PJ, Olkkola KT 1988 Oral activated charcoal in the treatment of intoxications. Role of single and repeated doses. Med Toxicol Adverse Drug Exp 3:33-58

15. McLuckie A, Forbes AM, Ilett KF 1990 Role of repeated doses of oral activated charcoal in the treatment of acute intoxications. Anaesth Intensive Care 18:375-384

16. Harris CR, Filandrinos D 1993 Accidental administration of activated charcoal into the lung: aspiration by proxy. Ann Emerg Med 22:1470-1473

17. Rau NR, Nagaraj MV, Prakash PS, Nelli P 1988 Fatal pulmonary aspiration of oral activated charcoal. [letter] BMJ 297:918-19

18. Elliot CG, Colby TV, Kelly TM, Hicks HG 1989 Charcoal lung: bronchiolitis obliterans after aspiration of activated charcoal. Chest 96:672-674 